\title{
Reduction in postsurgical adhesion formation after cardiac surgery in a rabbit model using N,0-carboxymethyl chitosan to block cell adherence
}

\author{
Juan Zhou, MD, PhD, ${ }^{a}$ Robert S. Liwski, MD, PhD, ${ }^{b}$ Clive Elson, $\mathrm{PhD}^{,}{ }^{\mathrm{c}}$ and Timothy D. G. Lee, $\mathrm{PhD}^{\mathrm{a}, \mathrm{b}}$
}

From the Departments of Surgery and Pathology, ${ }^{\mathrm{b}}$ Faculty of Medicine, Dalhousie University, Halifax, Nova Scotia; and Department of Chemistry, ${ }^{\mathrm{c}}$ Saint Mary's University, Halifax, Nova Scotia.

This work was supported in full by Kytogenics Pharmaceuticals Ltd. Dr Elson reports consulting fees, equity ownership, and employment by Kytogenics Pharma Ltd, the manufacturer of the gel described in this report. Drs Zhou, Liwski, and Lee have no financial interest in Kytogenics Ltd.

Received for publication Jan 18, 2007; revisions received Aug 23, 2007; accepted for publication Sept 19, 2007.

Address for reprints: Tim Lee, PhD, Sir Charles Tupper Building, 5850 College Street, Dalhousie University, Halifax, NS, Canada, B3H 4H7 (E-mail: tim.lee@dal.ca).

J Thorac Cardiovasc Surg 2008; 135:777-83 $0022-5223 / \$ 34.00$

Copyright $\odot 2008$ by The American Association for Thoracic Surgery

doi:10.1016/j.jtcvs.2007.09.033
Objective: The study objectives were to (1) assess the efficacy of N,O-carboxymethyl chitosan on postsurgical adhesion formation after cardiac surgery using a rabbit cardiac injury model and (2) explore the mechanism of action of N,O-carboxymethyl chitosan in the prevention of postsurgical adhesions using in vitro experimentation.

Methods: In the rabbit cardiac injury model, cardiac injury was generated by abrading the anterior surface of the heart with gauze and desiccated with oxygen. The rabbits were then either treated with N,O-carboxymethyl chitosan gel and solution on the injured surface or not treated. Fourteen days or 3 months after surgery, the severity and area of adhesion between the heart and sternum were evaluated. In the in vitro adherence assay, murine fibroblasts and macrophages were labeled with ${ }^{3} \mathrm{H}$-thymidine and added to sterile tissue culture plates that had been precoated with N,O-carboxymethyl chitosan solution, culture medium, or hyaluronic acid. After incubation, the cells adherent to the coated plates were harvested and the levels of ${ }^{3} \mathrm{H}$-thymidine were measured.

Results: Animals treated with N,O-carboxymethyl chitosan gel and solution showed significantly $(P<.01)$ reduced severity and area of adhesion formation. Murine fibroblasts and macrophages did not adhere to N,O-carboxymethyl chitosan-coated tissue culture plates, even in the presence of serum.

Conclusion: The application of N,O-carboxymethyl chitosan gel and solution significantly reduces the severity of postsurgical adhesion formation after cardiac surgery in the rabbit model. The inability of fibroblasts to adhere to N,O-carboxymethyl chitosan-coated surfaces suggests that N,O-carboxymethyl chitosan may act as a biophysical barrier.

$\mathrm{P}$ ostsurgical adhesion formation is a common complication of cardiac surgery. Adhesion formation is not only troublesome for secondary cardiac surgery, involving increased risks of heart injury and severe hemorrhage, but also may obliterate the pericardial space and constrict heart function. ${ }^{1}$ A product that can prevent adhesion formation after cardiac surgery would be of great benefit. A number of approaches to reduce or prevent adhesion formation have been examined in animal models, and some therapies have reached clinical trials and the marketplace. A common feature in a number of these interventions has been an attempt to establish a physical barrier between injured surfaces by the application of films or gels. ${ }^{2-4}$

A chitosan derivative, N,O-carboxymethyl chitosan (NOCC), has shown significant promise as an antiadhesion agent. A number of reports have provided evidence that this agent limits adhesion formation and adhesion reformation in animal abdominal injury models ${ }^{5-7}$ In addition to the preclinical evidence, NOCC was recently shown to reduce adhesion reformation in stage I/II clinical trials. ${ }^{8}$ In the animal models, the highest efficacy was achieved by applying NOCC gel, and subsequently NOCC solution, between surgically damaged surfaces rather than at sites distant to the injury. ${ }^{7}$ This suggests that NOCC functions as a physical barrier, but the mechanism of action remains unclear. 


$$
\begin{aligned}
& \text { Abbreviations and Acronyms } \\
& \begin{aligned}
\mathrm{dpm}= & \text { disintegrations per minute } \\
\mathrm{FCS}= & \text { fetal calf serum } \\
\mathrm{HA}= & \text { hyaluronic acid } \\
\mathrm{NOCC}= & \text { N,O-carboxymethyl chitosan } \\
\text { sfRPMI = } & \text { serum-free Roswell Park } \\
& \text { Memorial Institute [medium] }
\end{aligned}
\end{aligned}
$$

Adhesion formation is initiated by blood coagulation, followed by fibrin matrix formation and tissue remodeling. ${ }^{9,10}$ During these processes, fibroblast deposition on the fibrin matrix and production of matrix proteins, such as fibrin, fibronectin, and collagen, occur. Fibrin and fibronectin are major substratum for attracting the fibroblasts that, along with collagen, form the predominant components of postsurgical adhesions. ${ }^{11,12}$ Therefore, blocking fibroblast deposition could be an effective strategy to prevent adhesion formation.

In the present study, we first assess the effect of NOCC gel and solution on adhesion formation after cardiac surgery in a rabbit model. Second, we examine the mechanism of action of NOCC in the prevention of postsurgical adhesions in vitro experimentation. We demonstrate that application of NOCC products inhibits postsurgical adhesion formation after cardiac surgery. In vitro experiments demonstrate that NOCC inhibits the adhesion of fibroblasts and macrophages to plastic surfaces, a finding that is consistent with our hypothesis that NOCC acts as a physical barrier to prevent fibroblast and macrophage deposition and subsequent adhesion formation.

\section{Materials and Methods \\ Animals}

Female New Zealand White rabbits $(2.5-3.0 \mathrm{~kg}$ ) were purchased from Charles River, maintained in the Dalhousie University Faculty of Medicine animal care facility, and provided with food and water ad libitum. All animal experimentation was undertaken in compliance with the guidelines of the Canadian Council of Animal Care.

\section{Cell Lines}

Mouse 3T3 fibroblasts and mouse J774 macrophages were purchased from the American Type Culture Collection (Manassas, Va) and maintained in a $75-\mathrm{cm}^{2}$ tissue culture flask containing Roswell Park Memorial Institute [medium] (RPMI) culture medium (ICN Biomedicals, Aurora, Ohio) supplemented with $10 \%$ fetal calf serum (FCS), $20 \mathrm{mmol} / \mathrm{L}$ HEPES, $100 \mathrm{U} / \mathrm{mL}$ penicillin, 100 $\mu \mathrm{g} / \mathrm{mL}$ streptomycin, $2 \mathrm{mmol} / \mathrm{L}$ L-glutamine, and $50 \mu \mathrm{M}$ 2-mercaptoethanol (all from Life Technologies, Burlington, $\mathrm{ON}$ ). Cells were maintained at the density of $1 \times 10^{5}$ cells $/ \mathrm{mL}$ to $1 \times 10^{6}$ cells $/ \mathrm{mL}$ and were fed every 3 days by replacement with fresh culture medium.

\section{NOCC Solution and Hyaluronic Acid}

Sterile NOCC products, including a cross-linked $1 \%$ NOCC gel and a non-crosslinked $2 \%$ NOCC, solution were provided by Kytogenics Pharmaceuticals Ltd (Halifax, NS, Canada). Both gel and solution were used in a sterile manner in the cardiac injury model. For in vitro experiments, NOCC solution was diluted 1:20 in serum-free RPMI (sfRPMI) to achieve a final concentration of $0.1 \%$ (w/v). Hyaluronic acid (HA) was obtained from Sigma-Aldrich (Ontario, Canada) and prepared in sfRPMI to achieve a final concentration of $0.4 \%(\mathrm{w} / \mathrm{v})$ solution. Both $0.1 \%$ NOCC and $0.4 \%$ HA were prepared in a sterile manner.

\section{Cardiac Injury Model}

Rabbits were anesthetized with ketamine $(50 \mathrm{mg} / \mathrm{kg}$ ) and xylazine $(5 \mathrm{mg} / \mathrm{kg}$ ) intramuscular injection for induction, followed by endotracheal intubation and mechanical ventilation, and maintained with isoflurane $(3 \%)$. Cardiac activity was monitored with electrocardiography throughout the entire surgery. After preparation for aseptic surgery, a midline incision of skin and muscles was made by scalpel and electrocautery, respectively. Bleeding was controlled with electrocautery. The thorax was opened via a median sternotomy using straight surgical scissors, and then a sternal retractor was placed to obtain adequate exposure. The anterior pericardium was opened at approximately 4 to $5 \mathrm{~cm}$ in length to expose the anterior surface of the heart. The anterior surface of the heart $(\sim 2 \times 1 \mathrm{~cm})$ was abraded for 5 minutes with gauze (to develop punctuate bleeding) and desiccated twice (30 seconds each time) with oxygen at a flow rate of $5 \mathrm{~L} / \mathrm{min}$. After the injury, the animals were then either treated with NOCC gel and solution or not treated with any NOCC products (as control group). When NOCC gel and solution were applied, $5 \mathrm{~mL}$ of NOCC gel was placed on the injured surface of the heart and $2 \mathrm{~mL}$ of solution was applied around the gel. After treatment, leaving the pericardium open, the sternum was closed with interrupted 2-O Dexon sutures. The muscle and skin were closed in layers with 3-O Dexon and staples, respectively. Air was sucked from the chest via a tube placed just before closure of the sternum. All animals received analgesic (morphine $2.0 \mathrm{mg} / \mathrm{kg}$ intravenously) and antibiotics (enrofloxacin [Baytril, Bayer Healthcare, Montville, NJ] $5 \mathrm{mg} / \mathrm{kg}$, by subcutaneous injection) at the time of surgery and 2 additional doses of analgesic (buprenorphine, $0.05 \mathrm{mg} / \mathrm{kg}$ by subcutaneous injection) at 12 and 24 hours postsurgery. Animals recovered in heated cages, with frequent observation, and received food and water ad libitum.

At 14 days or 3 months after the surgery, rabbits were sacrificed by lethal injection of sodium pentobarbital $(340 \mathrm{mg} / \mathrm{mL})$ for assessment of poststernal adhesion formation (adhesions between the heart and the sternum). The anterior chest, including the ribs, sternum, and heart adherent to the sternum, was removed from rabbits. The posterior pericardium was opened, and the edge of adhesions between the heart and the sternum was marked with Indian ink. The severity of adhesions was evaluated by measuring the strength of adhesions. This was graded on a 5-point scale by pulling the heart from the sternum and evaluating how well the tissue could be separated: grade $0=$ no adhesions; grade $1=$ very weak adhesions that are easy to tear apart by hand; grade 2 = weak adhesions that can be dissected with fingers; grade $3=$ moderately strong adhesions that require partial dissection with scissors; grade $4=$ strong adhesions that require dissection with scissors; grade $5=$ very strong adhesions that require dissection with scissors. After removal of the heart from the sternum, the area of adhesion could be visualized by the Indian ink outline. These were photographed, and the areas of adhesion indicated by the markers were evaluated by a computerized digital image analysis. 
In all cases animals were randomly assigned to the treatment group or control group before surgery. The surgeon performing the operation was blinded to the assignment. All animals were ear tattooed by animal care personnel immediately before the operation. After the cardiac injury was complete, the NOCC gel and solution were applied by a technician who was aware of the treatment assignment and tattoo number but not otherwise involved in the operation. On the day of sacrifice, all animals were randomly presented for evaluation by the animal care staff. Evaluation of adhesions was performed by the operating surgeon. The surgical assistant noted the tattoo number and the evaluation. After all animals were evaluated, adhesion data, including the severity and area of adhesion, were provided to the treatment technician, who then matched the tattoo numbers recorded with the treatment applied and the adhesion data recorded.

\section{Adherence Assays}

Cell adherence to coated plastic was assessed using radioactive labeling techniques. Briefly, 3T3 fibroblasts or J774 macrophages were removed from the stock culture flask by incubating the cells with Hank's balanced salt solution containing $0.25 \%$ (w/v) trypsin and $0.1 \%(w / v)$ EDTA (Cellgro, Manassas, Va) at $37^{\circ} \mathrm{C}$ for 3 minutes and then by scraping the inner surface of the flask with a rubber cell scraper. The cell suspension was transferred into a $15-\mathrm{mL}$ tube and centrifuged at $200 g$ for 5 minutes. Cells were resuspended in sfRPMI and washed with sfRPMI twice by centrifugation. The cells were labeled with ${ }^{3} \mathrm{H}$-thymidine $(1 \mu \mathrm{Ci} / \mathrm{mL}$, ICN radiochemicals, Irving, Calif) in sfRPMI for 4 hours at $37^{\circ} \mathrm{C}$. After washing with sfRPMI, the cells were resuspended in 1 of the following 5 groups of media: (1) sfRPMI; (2) sfRPMI + 2\% FCS; (3) sfRPMI $+5 \%$ FCS; (4) sfRPMI + 10\% FCS; or (5) $100 \%$ normal mouse serum. Cells were then added to the precoated wells of 96-well tissue culture plates in 2 sets of triplicate wells (each triplicate set is plated twice) at a concentration of $2 \times 10^{4}$ cells/well. After 90 minutes of incubation at $37^{\circ} \mathrm{C}$ to allow cells to adhere to the plates, 1 set of the triplicate wells was washed with serum-free medium 3 times to remove nonadherent cells before harvesting. The other set of triplicate wells was not washed. Cells from both sets were then harvested onto glassfiber filtermats (Skatron) using an automatic cell harvester (Skatron, Sterling, Va). The ${ }^{3} \mathrm{H}$-thymidine levels were determined using a $\beta$-counter and were expressed as disintegrations per minute (dpm). The ratio of radioactivity of the first set of wells (adherent cells) to the second set of wells (nonadherent and adherent cells) was used to calculate the percent adhesion. Percent adhesion was calculated using the following formula: (dpm adherent cells/ dpm total cells) $\times 100$.

The wells of the plate were precoated with 1 of the 3 media: (1) sfRPMI; (2) $0.1 \%$ NOCC; or (3) $0.4 \%$ HA. Precoating was achieved by placing $100 \mu \mathrm{L}$ of sfRPMI or $0.1 \%$ NOCC or $0.4 \%$ $\mathrm{HA}$ in the wells followed by incubation at $37^{\circ} \mathrm{C}$ for 18 hours. The precoated wells were then washed with sterile phosphate-buffered saline $\left(0.15 \mathrm{~mol} / \mathrm{L} \mathrm{NaCl}, 0.05 \mathrm{~mol} / \mathrm{L} \mathrm{Na}_{2} \mathrm{PO}_{4} \mathrm{pH} 7.4\right)$ extensively to remove unbound NOCC or HA. Wells coated with serum free medium were used as controls only.

\section{Statistics Analysis}

The Wilcoxon rank-sum test was used to assess the data obtained from cardiac injury model because it did not follow a normal distribution. One-way analysis of variance was used to analyze the data obtained from in vitro experimentation.

\section{Results}

Application of NOCC Gel and Solution Significantly Reduced Adhesion Formation After Cardiac Surgery

All of the rabbits that underwent cardiac surgery survived the surgery and were maintained until sacrifice. At sacrifice, we only examined adhesions between the epicardium and the sternum. At sacrifice no gel or solution could be visualized in the thoracic cavity, indicating that the NOCC gel and solution had been completely eliminated by the time of sacrifice. Thus, no gel or solution was present to bias the blinded assessment of severity and size of the adhesions.

Twenty animals (10 NOCC treated and 10 controls) were sacrificed 14 days postsurgery. At the 14-day time point, all the animals developed postsurgical adhesions between the anterior surface of the epicardium and the posterior surface of the sternum. However, the severity of adhesions in NOCC-treated animals was significantly reduced compared with that in control animals. As shown in Table 1, 9 of 10 animals in the control group, which did not receive NOCC treatment, had strong adhesions (grades 4 and 5). These adhesions required sharp dissection to separate the heart from the sternum. In contrast, in the NOCC-treated group, only 1 of 10 animals showed strong adhesions (grade 4) and 1 of 10 showed moderate (grade 3) adhesions. Eight of 10 animals showed only weak and loose adhesions (grades 1 and 2), and these adhesions were easily separated with fingers. These data demonstrate

TABLE 1. Postsurgical adhesion formation 14 days after cardiac surgery

\begin{tabular}{llllllll}
\hline & \multicolumn{3}{c}{ NOCC treated } & & \multicolumn{3}{c}{ Control } \\
\cline { 2 - 4 } \cline { 6 - 7 } $\begin{array}{c}\text { Animal } \\
\text { No. }\end{array}$ & Severity & $\begin{array}{c}\text { Area } \\
\left(\mathbf{c m}^{2}\right)\end{array}$ & $\begin{array}{c}\text { Cumulative } \\
\text { score }\end{array}$ & & Severity & $\begin{array}{c}\text { Area } \\
\left(\mathbf{c m}^{2}\right)\end{array}$ & $\begin{array}{c}\text { Cumulative } \\
\text { score }\end{array}$ \\
\hline 1 & 4 & 2.24 & 8.96 & & 3 & 4.35 & 13.5 \\
2 & 1 & 3.29 & 3.29 & & 5 & 6.06 & 30.3 \\
3 & 1 & 4 & 4 & & 5 & 3.83 & 19.15 \\
4 & 1 & 1.97 & 1.97 & & 5 & 3.11 & 15.55 \\
5 & 2 & 3.38 & 6.76 & & 4 & 2.32 & 9.28 \\
6 & 1 & 0.89 & 0.89 & & 4 & 2.36 & 9.44 \\
7 & 3 & 2.73 & 8.19 & & 4 & 3.09 & 12.36 \\
8 & 1 & 2.18 & 2.18 & & 5 & 1.83 & 9.15 \\
9 & 1 & 2.33 & 2.33 & & 4 & 2.5 & 10.0 \\
10 & 2 & 1.67 & 3.34 & & 4 & 2.17 & 8.68 \\
Average: & $1.70^{*}$ & 2.47 & $4.19^{*}$ & & 4.30 & 3.16 & 13.74
\end{tabular}

NOCC, N,0-carboxymethyl chitosan. Severity of adhesion graded on a scale of 1 to 5 . Area of adhesions expressed as centimeters squared. Cumulative adhesion score is established by multiplying the severity of adhesions with the area of adhesions. * Significantly different $(P<.01$; Wilcoxon rank-sum test) from control. 
that NOCC treatment significantly reduces adhesion formation after cardiac surgery.

The area of adhesions between the heart and the sternum was obtained through computerized image analysis, and the results are shown in Table 1. These results indicate that there is no significant reduction of the average of adhesion area in NOCC-treated animals when compared with nontreated control animals.

A cumulative adhesion score for each animal, which was calculated by multiplying the adhesion grade and adhesion area, was used to evaluate the overall adhesion formation. These data are presented in Table 1. Statistical analysis confirmed that the average of the cumulative score of the NOCC-treated group is significantly $(P<.01)$ lower than that of the nontreated control group.

A second group of animals (7 NOCC treated and 5 controls) was sacrificed 3 months postsurgery. The data from these animals are presented in Table 2 . In contrast with the 14-day group in which only the severity was reduced, there was a highly significant reduction in both severity and area of adhesions in the NOCC-treated group. The cumulative score $(0.95)$ for the NOCC-treated group was significantly $(P<.001)$ lower than for the control group (9.59).

\section{NOCC Coating Prevents Fibroblasts and}

\section{Macrophages from Adhering to Coated Surfaces}

The data shown in Figure 1, $A$ demonstrate that when the culture wells were precoated with medium containing $0.1 \%$ NOCC, there was a significant reduction in the percentage of fibroblasts that adhered. A similar phenomenon with macrophages is shown in Figure 1, $B$. A highly significant $(P<.001)$ reduction of adherence was seen in NOCC-coated surfaces in comparison with the control,

TABLE 2. Postsurgical adhesion formation 3 months after cardiac surgery

\begin{tabular}{|c|c|c|c|c|c|c|}
\hline \multirow[b]{2}{*}{$\begin{array}{c}\text { Animal } \\
\text { No. }\end{array}$} & \multicolumn{3}{|c|}{ NOCC treated } & \multicolumn{3}{|c|}{ Control } \\
\hline & Severity & $\begin{array}{l}\text { Area } \\
\left(\mathrm{cm}^{2}\right)\end{array}$ & $\begin{array}{c}\text { Cumulative } \\
\text { score }\end{array}$ & Severity & $\begin{array}{l}\text { Area } \\
\left(\mathrm{cm}^{2}\right)\end{array}$ & $\begin{array}{c}\text { Cumulative } \\
\text { score }\end{array}$ \\
\hline 1 & 0 & 0 & 0 & 4 & 1.50 & 5.99 \\
\hline 2 & 4 & 1.19 & 4.76 & 4 & 1.86 & 7.43 \\
\hline 3 & 0 & 0 & 0 & 4 & 1.27 & 5.08 \\
\hline 4 & 0 & 0 & 0 & 5 & 1.41 & 7.05 \\
\hline 5 & 1 & 0.44 & 0.44 & 5 & 4.48 & 22.39 \\
\hline 6 & 2 & 0.22 & 0.44 & & & \\
\hline 7 & 2 & 0.51 & 1.02 & & & \\
\hline Average: & $1.29^{*}$ & $0.34^{*}$ & $0.95^{*}$ & 4.4 & 2.10 & 9.59 \\
\hline
\end{tabular}

NOCC, N,0-carboxymethyl chitosan. Severity of adhesion is graded on a scale of 1 to 5 . Area of adhesions expressed as centimeters squared. Cumulative adhesion score is established by multiplying the severity of adhesions with the area of adhesions. ${ }^{*}$ Significantly different $(P<.01$; Wilcoxon rank-sum test) from control.
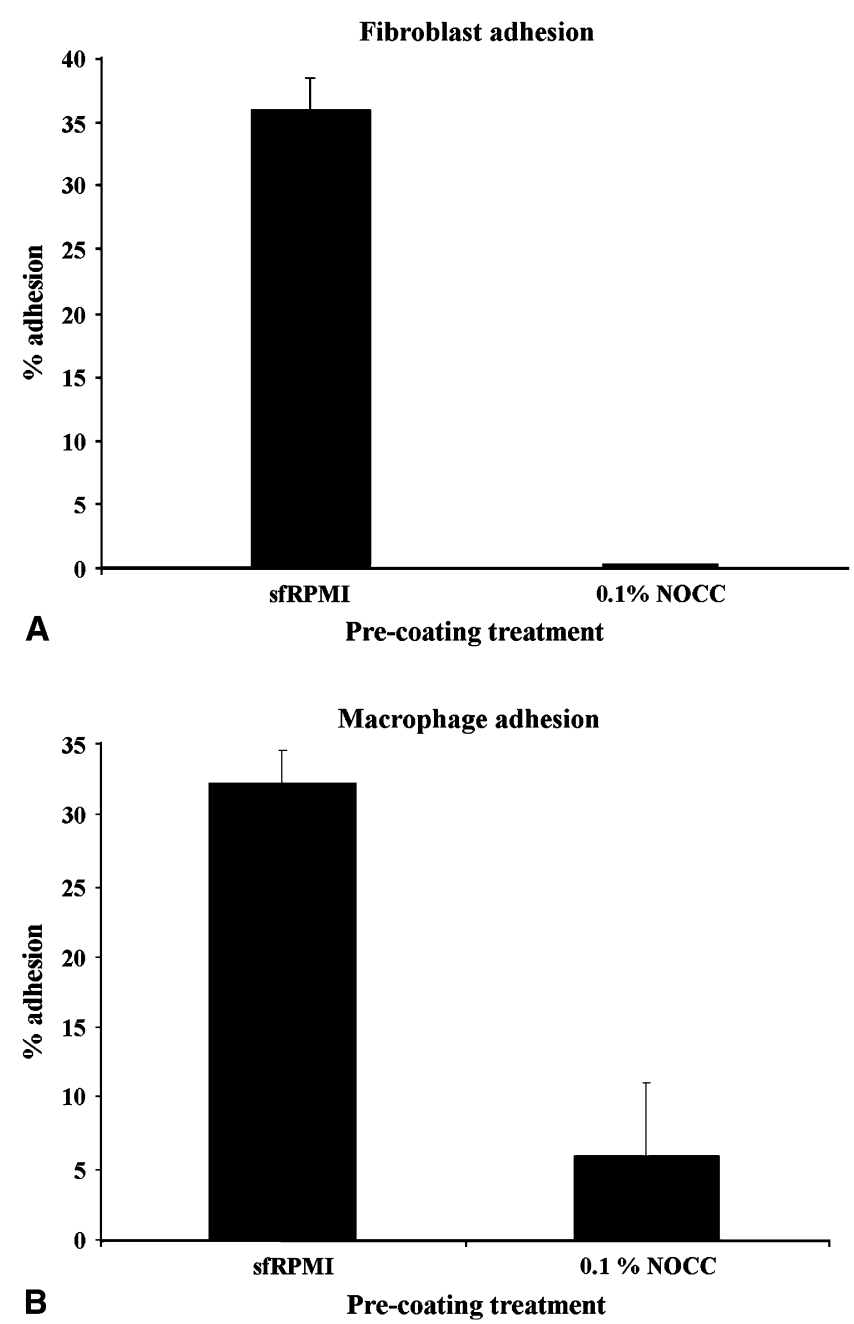

Figure 1. Precoating of plates with NOCC inhibits adhesion of $3 \mathrm{T3}$ fibroblasts (A) and $\mathbf{J 7 7 4}$ macrophages (B) to the coated plates in the ${ }^{3} \mathrm{H}$-thymidine-labeled adherence assay. The plates were precoated with sfRPMI or sfRPMI containing $0.1 \%$ NOCC. Data are expressed as mean \pm standard deviation of triplicate wells and are representative of four experiments. sfRPMI, Serum-free Roswell Park Memorial Institute [medium]; NOCC, N,0-carboxymethyl chitosan.

noncoated surfaces when both the ${ }^{3} \mathrm{H}$-thymidine assay and the ${ }^{51} \mathrm{Cr}$ assay (data not shown) were used.

NOCC Coating Prevents Fibroblasts from Adhering to the Coated Surface Even in the Presence of Serum The data in Figure 2 show that $32.7 \% \pm 0.2 \%$ of the fibroblasts plated in sfRPMI adhered to the wells that had been precoated with serum-free medium (the first bar from the left). As seen in the earlier experiments, cells plated in sfRPMI did not adhere to the NOCC-coated wells (second bar of histogram). The addition of FCS to the plating media 


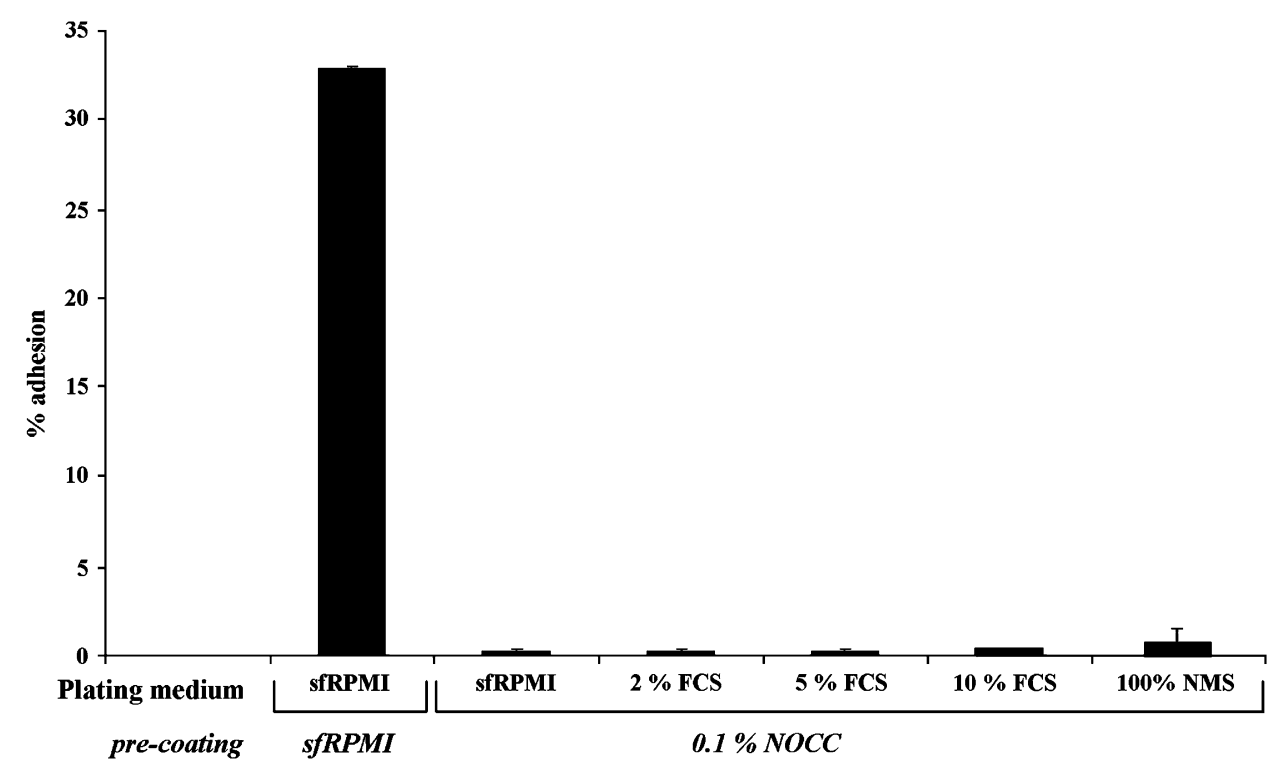

Figure 2. Precoating plates with NOCC blocks fibroblast adhesion regardless of the concentration of serum used in ${ }^{3} \mathrm{H}$-thymidine-labeled adherence assay. The plates were precoated with sfRPMI or sfRPMI containing $0.1 \%$ NOCC. The adhesion of fibroblasts, suspended in RPMI media containing varying concentration of FCS or suspended in mouse serum (100\% normal mouse serum), to the coated plates was measured by the ${ }^{3} \mathrm{H}$-thymidine assay. Data are expressed as mean \pm standard deviation of triplicate wells and are representative of 2 experiments. sfRPMI, Serum-free Roswell Park Memorial Institute [medium]; FCS, fetal calf serum; NMS, normal mouse serum; NOCC, N,O-carboxymethyl chitosan.

did not significantly $(P>.05)$ increase the adherence of fibroblasts to the coated wells. Even plating the fibroblasts in $100 \%$ homologous normal mouse serum did not significantly increase adhesion to the NOCC-precoated wells $(P>.05)$.

\section{NOCC Acts as a More Efficient Barrier to Cell} Adherence than Hyaluronic Acid

In the final experiment, we tested $0.4 \% \mathrm{HA}$ as a coating medium to prevent the adhesion of fibroblasts, in comparison with $0.1 \%$ NOCC solution. The results are shown in Figure 3, As expected, fibroblast adherence to sfRPMI-coated wells was enhanced if the $3 \mathrm{~T} 3$ cells were plated in pure mouse serum. Coating the wells with $0.4 \%$ HA significantly $(P<.05)$ reduced the adherence of cells to the plates. This effect of HA was lost if the cells were plated in mouse serum. In contrast, precoating wells with NOCC completely prevented adherence, and the presence of serum in the plating media did not ablate this adherent inhibition.

\section{Discussion}

In previous experiments we demonstrated that application of NOCC gel and NOCC solution between surgically injured surfaces significantly reduces postsurgical adhesion formation and adhesion reformation in a variety of animal models. ${ }^{5-7}$ Because blocking adhesion formation may inhibit normal wound healing, we examined the effect of NOCC on wound healing using a rat aorta anastomosis model, a rat large bowel anastomosis model, and a rat abdominal skin healing model. ${ }^{6}$ We demonstrated that normal healing occurred in the presence of NOCC, and that NOCC had no effect on infection at the wound site. ${ }^{6}$

In the present study, we assessed the ability of the NOCC gel and solution to prevent adhesion formation after cardiac surgery in a rabbit cardiac injury model. This is a preclinical model with significant predictive value for clinical use. In our experiments, cardiac activity of rabbits was closely monitored with electrocardiography throughout the entire surgery. This procedure allowed us to successfully complete the cardiac surgery without the loss of any animals during cardiac surgery. We selected day 14 postsurgery to evaluate the adhesion formation, an early stage of postsurgery. It has been suggested that the injured surfaces are most susceptible to adhesion formation between 16 and 36 hours postsurgery and that the presence of a physical barrier between injured tissue surfaces during this critical stage would minimize adhesion formation. ${ }^{13,14}$ In addition, we assessed adhesion formation at a more clinically relevant time point ( 3 months) to assess the longer term effects of the NOCC treatment.

The data obtained 14 days and 3 months after the cardiac surgery indicate that NOCC gel and solution significantly reduce the severity of retrosternal adhesion formation and the overall cumulative adhesion score. The data obtained at 


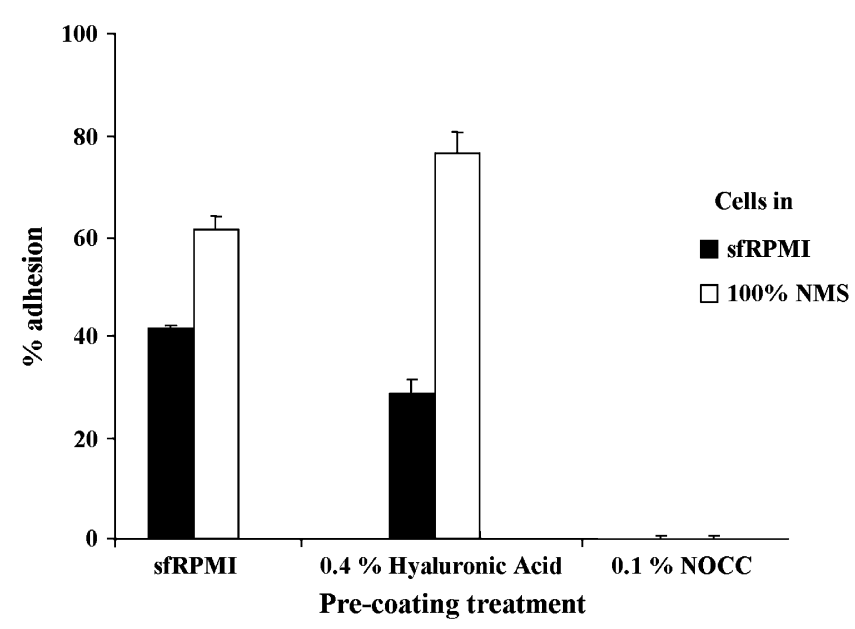

Figure 3. HA does not prevent adhesion of fibroblasts in the presence of homologous serum. Mouse fibroblasts were suspended in either sfRPMI or $100 \%$ normal mouse serum before plating on tissue culture plates that were precoated with sfRPMI, $0.4 \% \mathrm{HA}$, or $0.1 \%$ NOCC. Adherence to the plates was measured by ${ }^{3} \mathrm{H}$-thymidine adherence assay. Data are expressed as mean \pm standard deviation of triplicate wells and are representative of 2 experiments. sfRPMI, Serum-free Roswell Park Memorial Institute [medium]; NMS, normal mouse serum; NOCC, N,0-carboxymethyl chitosan.

3 months also indicated that NOCC treatment significantly reduces the adhesion area. These data suggest that NOCC treatment is effective in reducing retrosternal adhesion formation after cardiac surgery.

To investigate the mechanisms responsible for the effect of NOCC gel and solution on prevention of adhesion formation, we designed in vitro experiments. We hypothesized that NOCC prevents adhesion formation by creating a barrier to fibroblast and macrophage deposition at the site of injury. Adhesion formation begins with a fibrin matrix, typically associated with blood coagulation. This matrix is gradually replaced by granular tissue mainly containing fibroblasts and macrophages. Finally, the adhesion matures into a fibrous band and tissue remodeling occurs. ${ }^{9,10}$ During the process of adhesion formation, deposition of fibroblasts and macrophages in the matrix is critical. These cells produce critical components of the matrix and are involved in cell recruitment to the development of a fibrous band. ${ }^{11}$ Because the deposition of fibroblasts and macrophages is a critical step in adhesion formation, we designed experiments to examine whether NOCC reduces deposition of fibroblasts and macrophages on tissue culture surfaces in vitro.

We precoated the tissue culture plastic with $0.1 \%$ NOCC, effectively establishing a barrier between the cells and the adherent surface. The precoating virtually eliminated fibroblast adherence and reduced macrophage adherence by more than $80 \%$. The fact that the cells do not adhere to NOCC-coated surfaces strongly suggested a barrier effect for NOCC. Therefore, it is possible that NOCC functions in vivo to block fibroblast and macrophage attachment to the injured surfaces, thus interrupting fibrin matrix formation. The observed reduction of severity of adhesion formation without reduction in adhesion area after cardiac surgery in this rabbit model may be due to the reduced deposition of fibroblasts and macrophages in the developing lesions. Reduced deposition of fibroblasts and macrophages in these lesions would result in weaker adhesions. In fact, it is primarily the severity of the adhesion, not the extent of adhesion formation, that is significant for clinical cardiac surgery. Adhesions easily cleared by a surgeon's fingers will generally not affect the outcome of surgery.

It is not clear how the NOCC solution functions as a physical barrier to prevent fibroblasts from adhering to the coated plastic surface. Because NOCC is a negatively charged polysaccharide, it is possible that during coating, the NOCC tightly binds to the positively charged culture plate surface and then presents to the negatively charged cell membranes of the fibroblasts an inhospitable environment for adherence. Our earlier experiments (Clive Elson, $\mathrm{PhD}$, unpublished data; 2004) showed that NOCC adheres strongly to biological membranes and that NOCC would be expected to adhere tightly to significantly damaged surfaces on application.

It is of significant interest that NOCC solution prevents the deposition and adherence of fibroblasts onto surfaces even in the presence of extracellular matrix proteins such as vitronectin (in the serum-supplemented media). ${ }^{12}$ Fibronectin and vitronectin are 2 major extracellular matrix proteins abundant in serum. These proteins effectively facilitate the attachment of fibroblasts and macrophages to extracellular matrix by engagement of cell adhesion molecules on the cell surfaces. ${ }^{12,15,16}$ In this study, homologous serum enhances fibroblast adherence to tissue culture wells in vitro, probably reflecting the binding of fibronectin and vitronectin in the serum to the plate, which then facilitates cell adherence. However, even in the presence of these important serum proteins, fibroblast adherence is blocked if the wells are precoated with NOCC. Thus, in vivo, NOCC is probably not displaced from coated surfaces by these, and other, important serum proteins. This is a highly significant finding because serum proteins are abundant in the operative field. To be effective, NOCC must inhibit fibroblast and macrophage adherence in the presence of serum components.

In this study, we also examined HA because it is the basis of a number of products tested to reduce postsurgical adhesions. ${ }^{17-19}$ HA has also been shown to display significant efficacy in preventing postsurgical adhesions in animal models. ${ }^{6,20,21}$ We found that precoating surfaces with HA significantly reduced, but did not prevent, fibroblast adherence; however, this effect was eliminated by the presence of serum. This observation may explain why HA is ineffective in adhesion reformation ${ }^{20}$ and adhesion formation 
when it is applied after injury, ${ }^{21}$ because, in these situations, serum proteins are present in the injured area.

Taken together, our data demonstrate that NOCC products significantly reduce postsurgical adhesion formation after cardiac surgery. The reduced severity of adhesion formation results in adhesions that can be easily separated by fingers. This would yield significant benefits for clinical cardiac surgery if it proves true in clinical trials. In addition, NOCC effectively prevents fibroblast and macrophage adherence, even in the presence of homologous serum, suggesting that NOCC acts as a physical barrier in preventing postsurgical adhesion formation.

\section{References}

1. Macmanus Q, Okies JE, Phillips SJ, Starr A. Surgical considerations in patients undergoing repeat median sternotomy. J Thorac Cardiovasc Surg. 1975;69:138-43.

2. Okuyama N, Wang CY, Rose EA, Rodgers KE, Pines E, diZerega GS, et al. Reduction of retrosternal and pericardial adhesions with rapidly resorbable polymer films. Ann Thorac Surg. 1999;68:913-8.

3. Tsukihara H, Takamoto S, Kitahori K, Matsuda K, Murakami A, Novick RJ, et al. Prevention of postoperative pericardial adhesions with a novel regenerative collagen sheet. Ann Thorac Surg. 2006;81: 650-7.

4. Chorny M, Mishaly D, Leibowitz A, Domb AJ, Golomb G. Site-specific delivery of dexamethasone from biodegradable implants reduces formation of pericardial adhesions in rabbits. J Biomed Mater Res A. 2006;78: 276-82.

5. Kennedy R, Costain DJ, McAlister VC, Lee TD. Prevention of experimental postoperative peritoneal adhesions by N, O-carboxymethyl chitosan. Surgery. 1996;120:866-70.

6. Costain DJ, Kennedy R, Ciona C, McAlister VC, Lee TD. Prevention of postsurgical adhesions with $\mathrm{N}, \mathrm{O}$-carboxymethyl chitosan: examination of the most efficacious preparation and the effect of N, O-carboxymethyl chitosan on postsurgical healing. Surgery. 1997;121:314-9.

7. Zhou J, Elson C, Lee TDG. Reduction in post surgical adhesion formation and re-formation after abdominal surgery using N, O-carboxymethyl Chitosan. Surgery. 2004;135:307-12.
8. Diamond MP, Luciano A, Johns DA, Dunn R, Young P, Bieber E. Reduction of postoperative adhesions by N, O-carboxymethylchitosan: a pilot study. Fertil Steril. 2003;631-6.

9. diZerega GS. Contemporary adhesion prevention. Fertil Steril. 1994;61: 219-35.

10. Tamariz E, Grinnell F. Modulation of fibroblast morphology and adhesion during collagen matrix remodeling. Mol Biol Cell. 2002;13: 3915-29.

11. Clark RAF. Wound Repair, Overview and General Considerations. The Molecular and Cellular Biology of Wound Repair. 2nd ed. New York: Plenum Press; 1996.

12. Singer II, Scott S, Kawka DW, Kazazis DM, Gailit J, Ruoslahti E. Cell surface distribution of fibronectin and vitronectin receptors depends on substrate composition and extracellular matrix accumulation. J Cell Biol. 1988;106:2171-82.

13. Ricketts SA, Sibbons PD, Green CJ. Quantitative analysis of the development of experimentally induced post surgical adhesions: a microstereological study. Int J Exp Pathol. 1999;80:325-34.

14. Harris ES, Morgan RF, Rodeheaver GT. Analysis of the kinetics of peritoneal adhesion formation in the rat and evaluation of potential antiadhesive agents. Surgery. 1995;117:663-9.

15. Miyamoto S, Katz BZ, Lafrenie RM, Yamada KM. Fibronectin and integrins in cell adhesion, signaling, and morphogenesis. Ann N Y Acad Sci. 1998;857:119-29.

16. Sommerfeldt DW, McLeod KJ, Rubin CT, Hadjiargyrou M. Differential phosphorylation of paxillin in response to surface-bound serum proteins during early osteoblast adhesion. Biochem Biophys Res Commun. 2001; 285:355-63.

17. Johns DB, Rodgers KE, Donahue WD, Kiorpes TC, diZerega GS. Reduction of adhesion formation by postoperative administration of ionically cross-linked hyaluronic acid. Fertil Steril. 1997;68:37-42.

18. Rodgers KE, Johns DB, Girgis W, Campeau J, diZerega GS. Reduction of adhesion formation with hyaluronic acid after peritoneal surgery in rabbits. Fertil Steril. 1997;67:553-8.

19. Lundorff P, van Geldorp H, Tronstad SE, Lalos O, Larsson B, Johns DB, et al. Reduction of post-surgical adhesions with ferric hyaluronate gel: a European study. Hum Reprod. 2001;16:1982-8.

20. Urman B, Gomel V. Effect of hyaluronic acid on postoperative intraperitoneal adhesion formation and reformation in the rat model. Fertil Steril. 1991;56:568-70.

21. Urman B, Gomel V, Nazma J. Effect of hyaluronic acid on postoperative intraperitoneal adhesion formation in the rat model. Fertil Steril. 1991; 56:563-7. 